\title{
1 First Observations of Tracking Clouds Using Scanning ARM
}

Cloud Radars. 


\section{Abstract}

23 Tracking cloud entities using scanning cloud radars can help to document the temporal

24 evolution of cloud properties well before large drop formation ("first echo"). These

25 measurements compliment cloud and precipitation tracking using geostationary satellites

26 and weather radars. Here, two-dimensional (2-D) Along-Wind Range Height Indicator

27 (AW-RHI) observations of a population of shallow cumuli (with or without precipitation)

28 from the 35-GHz scanning ARM cloud radar (SACR) at the DOE Atmospheric Radiation

29 Measurements (ARM) program Southern Great Plains (SGP) site are presented.

30 Observations from the ARM SGP network of scanning precipitation radars are used to

31 provide the larger scale context of the cloud field and to highlight the advantages of the

32 SACR to detect the numerous, small, non-precipitating cloud elements. A new Cloud

33 Identification and Tracking Algorithm (CITA) is developed to track cloud elements. In

34 CITA, a cloud element is identified as a region having a contiguous set of pixels

35 exceeding a preset reflectivity and size threshold. The high temporal resolution of the

36 SACR 2-D observations $(30 \mathrm{sec})$ allows for an area superposition criteria algorithm to

37 match cloud elements at consecutive times. Following CITA, the temporal evolution of

38 cloud element properties (number, size, maximum reflectivity) is presented. The vast

39 majority of the designated elements during this cumulus event were short-lived non-

40 precipitating clouds having an apparent lifecycle shorter than 15 minutes. The advantages

41 and disadvantages of cloud tracking using a SACR are discussed. 


\section{1. INTRODUCTION}

44 Clouds play a critical role in Earth's climate system through their participation in

45 Earth's radiation budget, the hydrological cycle and the vertical redistribution of energy

46 and moisture in the atmosphere (e.g., Stephens 2005; Feingold and Seibert 2009). The

47 accurate representation of the factors that control cloud microscale and macroscale

48 properties in global climate models (GCMs) and cloud resolving models (CRMs) remains

49 a major challenge (e.g., Ghan et al. 1999; Grenier and Bretherton 2001; Park and

50 Bretherton 2009; Stevens and Feingold 2009). Continuously operating ground-based

51 supersites (Stokes and Schwartz 1994; Ackerman and Stokes 2003; Illingworth et al.

52 2007) equipped with a wide range of active and passive sensors provide detailed

53 information on cloud dynamical and microphysical properties. Until recently, the cloud

54 properties retrieved at these ground-based supersites were limited to the column sampled

55 by profiling sensors. Now, scanning cloud and precipitation radars are deployed to

56 provide information on the 3D structure of clouds and precipitation (Mather and Voyles

57 2013; Kollias et al. 2013a). One of the main scientific drivers for deploying scanning

58 cloud radars is the desire to document individual cloud elements as they transit through

59 different stages of their lifecycle (e.g., cloud formation, precipitation onset, dissipation).

60 Relating the temporal evolution of cloud systems to aerosol and large-scale meteorology

61 conditions could lead to a better understanding of the controls on low-clouds and

62 associated statistics.

63 Monitoring the temporal evolution of shallow cumulus clouds can be

64 accomplished using ground-based and airborne-based radar systems (multiple passes).

65 Capturing the early stage of cumulus development/detection (first echo) depends on the 
66 sensitivity of the radar system. When $\mathrm{cm}$-wavelength radars have been tasked for these

67 studies, the first echo coincides with the early development of small precipitation

68 particles (Knight and Miller 1993; Knight et al. 2002; Göke et al. 2007; Burnet and

69 Brenguier 2010). This early development of a precipitation echo implies that an efficient

70 collision-coalescence process drives particle growth in warm clouds. French et al. (1999)

71 used multiple passes over shallow cumulus clouds and observations from an airborne

$72 \mathrm{~mm}$-wavelength radar to document the temporal evolution of non-precipitating cumulus

73 clouds. These early efforts demonstrate the potential of scanning radars to monitor the

74 temporal evolution of shallow cumuli. However, the studied dataset is limited and, in the

75 majority of the studies, the use of cm-wavelength radars does not permit the

76 documentation of the cloud lifecycle before the development of small raindrop particles.

77 The spatial and temporal resolution of geostationary satellites also limits their

78 applicability for the detection of small, non-precipitating cumuli clouds.

79 The deployment of continuously operating scanning cloud radars (Mather and

80 Voyles 2013; Kollias et al. 2013a) at the US Department of Energy (DOE) Atmospheric

81 Radiation Measurement (ARM) program fixed and mobile sites offers the required

82 observational capabilities for monitoring the entire lifecycle of shallow cumuli clouds

83 over an extensive period of time. This is particularly germane for the ARM Southern

84 Great Plain (SGP) facility that is equipped with a distributed, multi-frequency scanning

85 radar network. This network includes a Scanning ARM Cloud Radar (SACR) with

86 sensitivity ( -30 dBZ at $10 \mathrm{~km})$ and spatial $(45 \mathrm{~m})$ and temporal resolution ( $\sim 30 \mathrm{sec}$ per

87 horizon-to-horizon scan) sufficient for continuous tracking of non-precipitating short-

88 lived cloud elements. 
89 Here, we present the first set of observations from this scanning cloud radar 90 facility during a warm season cloud event with a wide distribution of cloud types from 91 short-lived, non-precipitating cumuli to shallow, light precipitating cumulus clouds. The

92 details of a Cloud Identification and Tracking Algorithm (CITA) suitable for monitoring

93 the evolution of shallow cumulus in a Range-Height Indicator (RHI) plane are presented.

94 The detection capabilities and observed cloud statistics are compared to those obtained 95 from the scanning ARM precipitation radars. Preliminary statistics of the temporal 96 gradient of the radar reflectivity in shallow non-precipitating clouds are presented.

97 Finally, the limitations and capabilities of the ARM SGP facility to study the lifecycle of 98 cloud elements are discussed.

99 


\section{OBSERVATIONS}

101 The observations presented for this study were collected during the Midlatitude

102 Continental Convective Clouds Experiment (MC3E) conducted in April-June 2011 at the

103 ARM SGP facility. MC3E was the result of a collaborative effort between the DOE -

104 ARM program and its Climate Research Facility and the National Aeronautics and Space

105 Administration's (NASA) Global Precipitation Measurement (GPM) mission Ground

106 Validation (GV) program. The MC3E campaign was the first major field experiment

107 conducted at an ARM site after the acquisition of the new scanning ARM radar (Fig. 1,

108 Mather and Voyles 2013). The backbone infrastructure of the ARM SGP radar facility is

109 a distributed, heterogeneous network of profiling and scanning radar systems suitable for

110 the mapping of cloud and precipitation in 3D along with a small network of radiometers

111 and lidars. The SGP radar facility includes a 5.4-GHz (5.5 cm wavelength) C-band

112 Scanning ARM Precipitation Radar (C-SAPR), a network of three 9.4-GHz $(3.2 \mathrm{~cm}$

113 wavelength) X-band Scanning ARM Precipitation Radars (X-SAPR), and a dual-

114 frequency 35.3/93.9-GHz (8.5/3.2 mm) Scanning Cloud Radar (Ka-/W- SACR - Fig. 1).

115 The bulk of the observations presented in this manuscript are from the SACR

116 located in the Central Facility (CF). A Total Sky Imager (TSI), radiosonde launch

117 facility, 2-dimensional video disdrometer, wind profiler, and a laser ceilometer are also

118 present at the CF and are used in this study. The C-SAPR is located approximately $25 \mathrm{~km}$

119 to the north of the SGP-CF and the three X-SAPR systems are located in a triangular

120 configuration having a side (baseline) of approximately $20 \mathrm{~km}$ and centered on the SGP-

121 CF (Fig. 1). The primary motivation for the C-SAPR polarimetric radar system is to

122 provide the mesoscale context of precipitation over a $100-120 \mathrm{~km}$ domain range around 
123 the CF. The acquisition of the X-band radar network at the ARM SGP radar facility is

124 based on the desire to bridge the observational gap in sensitivity and spatial scales

125 between the dual-frequency scanning cloud radar and C-band polarimetric radar. The

126 SACR is a dual frequency scanning Doppler and polarimetric radar. However, during the

127 MC3E only the 35-GHz (Ka-band) radar frequency was operational (Table 1). With

128 sensitivity close to $-30 \mathrm{dBZ}$ at $10 \mathrm{~km}$ during nominal scanning parameters, the Ka-SACR

129 is capable of detecting clouds from their early formation stages. SACR scan strategies for

130 this event included horizon-to-horizon Along-Wind scans (AW-RHI), which requires the

131 primary wind direction at cloud level as an input. Once the wind direction is designated,

132 the cloud radar is expected to capture the evolution of the same cloud element as it is

133 advected over the instrument. For this particular case, the wind direction was determined

134 by consulting the relevant 1730 UTC radiosonde, wind profiler and visible satellite

135 imagines available in real time by the authors in the field. This wind direction was

136 visually confirmed in-situ by the authors and later corroborated by the 2030 UTC

137 radiosonde also launched at the CF. The scan direction was fixed for the duration of the

138 Ka-SACR AW-RHI scan strategy period. This was not a major concern since, during this

139 2.5-hour interval, wind in the cloud layer did not have an appreciable change in time or

140 height and there was not a distinguishable shear that could cause clouds to move

141 differently at different heights (Fig. 2). Furthermore, later inspection of the X-and C-

142 SAPR data showed that the motion field of clouds detected by these systems did not

143 differ substantially from the previously assumed flow (not shown). Once the wind

144 direction was determined, the SACR azimuth was aligned to this mean cloud layer wind

145 direction and the radar was tasked to perform long sequences of horizon-to-horizon AW- 
146 RHI scans to capture the evolution of the same cloud elements as they propagate towards,

147 over and away from the SACR. Additional details on the first generation of SACR

148 operational strategies and data post-processing are described in Kollias et al. (2013a, b).

149 Figure 3 demonstrates the advantage of using a heterogeneous network of radars

150 to document the temporal and spatial distribution of clouds from their early, low

151 reflectivity stages to their more mature precipitation-associated regime and following

152 lower reflectivity decay stage. Here an example of data collected by the Ka-SACR, C-

153 SAPR and the SE X-SAPR at a time for which primarily weak, non-precipitating clouds

154 were present over SGP-CF is shown. The Total Sky Imager (Fig. 3a) confirms the

155 presence of shallow, broken cumuli over the CF. These same clouds are observed by the

156 Ka-SACR overhead (Fig. 3d). All ARM radars observe a precipitating shallow cumulus

157 at a 5-10 km range from the Ka-SACR (southeast part of the AW-RHI scan). However,

158 the SAPRs have difficulty detecting the non-precipitating clouds observed by the Ka-

159 SACR illustrating the importance of millimeter radar observations for capturing shallow

160 non-precipitating clouds as well as the early stages of cloud evolution (Fig. 3). This is not

161 only due to differences in wavelength but also, in a smaller manner due to beam width,

162 relative cloud-to-radar distance and scanning strategy [Add References]. 


\section{3. CLOUD IDENTIFICATION AND TRACKING ALGORITHM}

165 The literature provides many examples of studies that have sought to follow the

166 evolution of cloud systems, with the most salient examples considering the lifecycle and

167 morphology of deep convective systems through the use of cloud-tracking algorithms

168 (e.g., Williams and Houze 1987; Velasco and Fritsch 1987; Rosenfeld 1987; Johnson et

169 al. 1998; Dixon and Wiener 1993; Machado et al. 1998). Satellite-based cloud tracking

170 studies identify deep convective cloud elements using infrared temperature $\left(T_{I R}\right)$

171 thresholds (e.g., Maddox 1980; Williams and Houze 1987; Chen et al. 1996) and

172 additional spatial coherency constraints (e.g., Machado et al. 1998; Futyan and Del Genio

173 2007). From the surface, radar-based cell designation and tracking algorithms capitalize

174 on radar reflectivity factor patterns and additional size constraints (e.g., Dixon and

175 Wiener 1993; Rosenfeld 1987; Johnson et al. 1998). These radar-based 'cell'

176 identification efforts then act as input for tracking algorithm components that analyze the

177 evolution of these cell patterns by determining area superposition between consecutive

178 time steps (e.g., Williams and Houze 1987; Boer and Ramanathan 1997; Machado et al.

179 1998), cloud propagation speed and superposition (Rosenfeld 1987; Johnson et al. 1998;

180 Futyan and Del Genio 2007), or by minimizing a cost function based on position and

181 element volume differences at consecutive times (e.g., Dixon and Wiener 1993). For such

182 deep convective cells and larger convective system examples, automatic and

183 semiautomatic (manual selection of the optimal candidate) tracking algorithms often

184 arrive at similar results (e.g., Machado et al. 1998).

185 The Cloud Identification and Tracking Algorithm (CITA) is developed to analyze

186 shallow cumulus clouds as they transit through different stages of their lifetime. The 
187 input to CITA is 2-D (range-height) Ka-SACR observations collected during an AW-RHI 188 scan (e.g., Fig. 3d). Range gates that contain no meteorologically significant detections 189 have been removed using a SNR threshold technique (e.g., Kollias et al. 2013b).

190 Furthermore, conditional sampling using the Linear Depolarization Ratio (LDR) and

191 radar reflectivity from the Ka-SACR, as well as the cloud base height from a ceilometer,

192 has been applied to classify and filter radar echoes associated with insects (Kollias et al.

193 2013b). Once these non-meteorological radar returns are removed, each AW-RHI radar

194 image is processed and CITA identifies a cloud element as those echoes having a 195 contiguous set of pixels with reflectivity greater or equal than $-50 \mathrm{dBZ}$ and assign them 196 an identification number (ID). The reflectivity of $-50 \mathrm{dBZ}$ matches the Ka-SACR 197 sensitivity at a $1-\mathrm{km}$ range during nominal scanning operational conditions. Although the 198 Ka-SACR will not be able to detect such weak cloud echoes at longer distance from the 199 radar, it is known that the Ka-SACR still offers sufficient sensitivity to observe weak, 200 non-precipitating clouds at extended range. To eliminate spurious echo clusters (due to 201 imperfect removal of radar noise-only range gates and insect returns), only those radar 202 echo clusters having areal coverage larger than $0.5 \mathrm{~km}^{2}$ are considered as cloud elements.

203 The second step within CITA is to apply a superposition criterion to track the 204 temporal-spatial movement of each ID assigned cloud element (Fig. 4b-c). The 205 superposition criterion identifies clusters that have the largest areal overlap in 206 consecutive radar scans and links them as echoes coming from the same cloud. This 207 assumption is considered reasonable for the AW-RHI SACR scans that were generated 208 every 30 seconds during this campaign. When two cloud elements merge, the larger 209 element is considered to continue and the smaller to terminate. Similarly, when a cloud 
210 element splits, the larger element continues with the previously assigned tracking ID and

211 the smaller appears as a new element. Validation for merges and splits detected by CITA

212 was done by time coherency in the range-height plane (this was determined by the

213 authors by visually inspecting every RHI scan from the SACR) and areal thresholds to

214 mitigate the weakest cloud features that may result from poor cumulus cloud RHI slicing.

215 An example application of CITA for a sequence of three consecutives along-wind

216 scans from Ka-SACR on 25 May 2011 is shown in Fig. 4. At the first time step, six cloud

217 cells are identified by CITA (ID: 1-6). Cloud elements having the tracking IDs 1 and 4

218 demonstrate an undisturbed lifecycle with no merges or splits during the provided

219 sequence (Fig. 5), whereas cloud elements assigned the IDs 2 and 3 (Fig. 4a) merge into a

220 single cloud element assigned to tracking ID 2 (Fig. 4b), and cloud ID 6 (Fig. 4b) splits

221 into two cloud elements with IDs 6 and 7 (Fig. 4c). A more in depth analysis of this

222 complex time sequence is shown later in this section. For this observing period, the

223 aforementioned criteria were applied for the large majority of the cases successfully, as

224 confirmed by visual inspection by the authors. This success of the echo overlap criteria

225 eliminates the need to explore more computationally demanding approaches that require

226 the estimation of the propagation speed, or the minimization of a cost function based on

227 position and volume to assess the best possible match for every cloud element. Several

228 tests were performed to evaluate the robustness of CITA results. Firstly, identified cloud

229 elements and associated evolution were manually inspected and verified by the authors.

230 Secondly, a simple test of algorithm repeatability was performed, the CITA approach was

231 applied to this dataset in reverse temporal order, with the CITA demonstrating very

232 similar ID counts and tracking results. Thirdly, the sensitivity of CITA to different 
233 detection thresholds was also tested for this event. The analysis indicated that for

234 reflectivity thresholds between -40 and $-50 \mathrm{dBZ}$, there was no significant change with the

235 number of clouds detected by the CITA approach or in the associated cloud primary

236 microphysical or geometrical proprieties (Fig. 5). However, if more restrictive threshold

237 changes were applied (e.g., higher reflectivity thresholds were selected), the impact on

238 tracking and evolution behaviors became more noticeable, as anticipated in light of past

239 radar and satellite tracking studies. One noteworthy consideration for the feedback

240 between reflectivity thresholds and CITA results was found when exploring the

241 implication of higher reflectivity thresholds on the documentation of the maximum cloud

242 element reflectivity. Specifically, single cloud elements often exhibit multiple maxima

243 regions, most likely attributed to coherent precipitation shafts that are embedded within

244 lower regions of cloud element reflectivity. When more restrictive thresholds (closer to

245 classical values for the presence of drizzle particles $\sim-10 \mathrm{dBZ}$ ) are applied, these multi-

246 core cloud elements are often reclassified into unique cloud entities rather than grouped

247 as a single cloud element. Since our study emphasizes the analysis of individual cloud

248 elements regardless of the number of interior precipitation cores, the behaviors associated

249 with thresholds closer to $-50 \mathrm{dBZ}$ seem to be the most appropriate to track singular cloud

250 features. However, this low reflectivity threshold can also presents challenges in the

251 interpretation of the output from CITA. Further inspection of a longer time sequence of

252 the clouds shown in figure 6 shows how complex the identification and tracking

253 algorithm can really be. As an example, when considering previous time steps, it can be

254 seen that IDs 2, 4 and 6 developed with their top capped at $2.5 \mathrm{~km}$ as early as 19:40UTC.

255 These cloud elements then individually merged with other cloud elements that originate 
256 later in time. These higher clouds present a cloud-top height at around $4.5 \mathrm{~km}$ and seem

257 to have a vertically extent of $1 \mathrm{~km}$ approximately (as can be seen from figure $4 \mathrm{c}$ ). The

258 problem with the low reflectivity threshold is that later in time these cloud elements tend

259 to separate, or split, again, and what one could interpret as a cloud that developed on top

260 could be analyzed as a two cloud entities that merged and later split, but if a more

261 restrictive threshold is applied then some clouds might not outlive the size threshold and

262 loose their initiation and/or decay moments and some of the statistics will be biased

263 towards larger and deep clouds. Therefore, it is a trade-off between fully capturing cloud

264 entities (and their edges and 'deeper cells' embedded in them as part of it and not

265 separate entities) and being able to perfectly and unambiguously distinguish between

266 different cloud entities at every time step in an automatic way. We believe that this type

267 of analysis and algorithm, if wants to be used independently of the case and in an

268 automated way, should be used with a large enough dataset and in a statistical way to

269 smooth out the possible biases introduced by the chosen reflectivity threshold since it was

270 shown that, in a statistical way the main variables analyzed here are not very sensitive to

271 the selected threshold (Fig. 5).

272

273 


\section{4. RESULTS}

275 Using Ka-SACR observations from the 25 May 2011, CITA identified a total of

2761323 individual cloud elements, 49 of them $(3.7 \%)$ were the result of a merge and 50

277 (3.78\%) of a split, and tracked the lifecycle of 338 distinct cloud elements (Fig. 6).

278 Therefore, the lifecycle of the vast majority of the cloud elements identified and tracked

279 by CITA mainly correspond to undisturbed and continuous evolution of cloud elements

280 with only one unique link at each time step of their lifecycle. In particular, three long-

281 lived shallow cloud elements (having cloud tops below $5 \mathrm{~km}$ ) tracked by the Ka-SACR

282 CITA during this period persisted for more than 25 minutes. These clouds attained

283 maximum reflectivity values exceeding $20 \mathrm{dBZ}$ during most of the observed cloud

284 lifecycle and exhibited cross-sectional areas in excess of $40 \mathrm{~km}^{2}$ (Fig. 6). The majority of

285 the shallow cloud elements observed however, were short-lived features with CITA

286 tracking lifecycles under 10 minute and low maximal reflectivity cores (below -5 dBZ,

287 see Figs. 6 and 7). Most often, weaker cloud elements are observed to have dissipated (or

288 exited the domain) after less than 5 minutes of their first detection. The validity that these

289 features are legitimate scans from shallow cloud elements (separate from 'detrained'

290 cloud elements in a sheered flow) was confirmed by author in-field observations and

291 sounding evidence, surface TSI camera imagery, as well as the absence of stronger

292 echoes in the SAPRs imagery near the cloud radar scanning transect during most periods

293 of observation.

294 It is important to note that there is a likely underrepresentation in these statistics

295 due to the radar scans not sampling the center of the cloud. Jorgensen et al. 1985 found a

296 diameter bias of approximately $22 \%$ when sampling spherical updraft cores from aircraft. 
297 This circular shape assumption for cumulus clouds may be applicable under low shear

298 conditions (such as the ones present during most of the time in this event, e.g. fig.2)

299 however; it might not be applicable for all the cloud lifecycle. Here we extended this

300 analysis to a generic ellipsoidal shape of cloud elements. As expected, biases for more

301 elongated cloud are more pronounced. For example, if clouds are elongated along the

302 wind direction having an axis ratio (major to minor dimension) of 0.8 , the expected bias

303 in areal coverage would be roughly $32 \%$. This suggests that future scan strategies should

304 include low-level PPI scans to effectively capture the structure of clouds and help

305 establish the placement of future AW-RHI.

306 The distribution of the maximum radar reflectivity values determined for all 338

307 cloud elements detected within the Ka-SACR sampling period is offered in Fig. 7a. This

308 plot indicates that the majority of the cloud elements attain a maximum radar reflectivity

309 between -20 to $-10 \mathrm{dBZ}$ (Fig. 7a). This magnitude of radar reflectivity at the SGP

310 location in central Oklahoma is consistent with clouds that do not produce drizzle ( $\mathrm{Lu}$ et

311 al., 2008). The frequency distribution of maximum horizontal-height area coverage

312 attained by all cloud elements peaks at the smallest-possible detectable area coverage for

313 CITA methods $\left(0.5 \mathrm{~km}^{2}\right)$.

314 Additional geometrical properties for the identified cloud elements are also 315 documented by CITA as a function of time. These parameters include the number of

316 cloud elements, the cloud element top height and the maximum horizontal length of the

317 cloud elements. The behaviors of these fields observed by the Ka-SACR for the 25 May

318 event are provided in Fig. 8. During times of precipitation in the vicinity of the cloud

319 radar (approximately 1920 UTC, 1950 UTC and 2040 UTC, Fig. 8e disdrometric 
320 observations at SGP - CF and C-SAPR estimations in a larger domain), there are a few

321 cumulus cloud elements (Fig. 8b) with extended horizontal lengths (Fig. 8c) and higher

322 relative top heights (Fig. 8d). In contrast, there is a suggestion of a strongly bimodal or

323 occasionally more complex distribution of cloud-top heights, most having shorter

324 cumulus horizontal length scales, within the non-precipitating and weaker initiating

325 times. During these sequences that include times at the beginning of the observation

326 period, one can consistently observe clouds having tops ranging from the lower levels

327 around $1.5 \mathrm{~km}$ (in association with the top of the boundary level) up to higher cloud top

328 levels near $3.5 \mathrm{~km}$ (in association with the freezing level), within the same scan. Yet,

329 when considering the periods associated with the onset or nearby precipitation, the

330 complexity of these tracked parameters is often reduced and cloud tops below $1.5 \mathrm{~km}$

331 disappear letting it mainly characterized by cloud elements with elevated tops. A

332 plausible explanation for this distribution relates to the evolution of the cloud field and its

333 associated dynamics. This event started with exclusively shallow cumulus clouds that

334 later transitioned to congestus clouds with some shallow cumulus still present in the

335 region. Therefore, the multilevel cloud top structure is likely to be a combination of very

336 shallow, non-precipitating mode, with some deeper precipitating cumulus with

337 entrainment at multiple levels. However, it is likely that in times when congestus clouds

338 dominate the near vicinity of the radar (approximately 1920 UTC, 1950 UTC and 2040

339 UTC) its associated cold pool-type outflow (noticeable from the drop in equivalent

340 potential temperature at SGP - CF, Fig. 8c) effectively act in a capacity to deter lower-

341 topped shallow, surface forced convection and temporarily reduces those observations for

342 an extended windows of atmospheric recovery. 
As a preliminary attempt to explore time-evolving cloud maximum and median

344 properties and the associated local rate of change (as potentially related to cloud

345 microphysical process and cloud growth and decay therein) the evolution of cloud radar

346 reflectivity fields from discrete shallow, non-precipitating cloud target examples are

347 provided in Fig. 9. To ensure these discrete, non-precipitating cumuli conditions, the

348 maximum and median parameter calculations and associated rate of change estimates are

349 limited to only those calculations from the individual cloud elements that persist for a

350 minimum of 5 minutes and have a maximum radar reflectivity that does not exceed -5

351 dBZ during the CITA cloud lifecycle tracking. As an additional constraint, we restrict the

352 dataset to only those pure or discrete cloud elements for which the CITA IDs have not

353 experienced a merge or a split. Finally, the remaining clouds are checked to ensure that a

354 maximum in the radar reflectivity factor in time occurs at least three time steps after

355 (before) the initial (final) detection by CITA. This latter constraint is intended to mitigate

356 the inclusion of clouds that either initiate too close to the edge of the Ka-SACR scanning

357 domain and might propagate out of the domain before achieving a mature state or mature

358 clouds entering the edge of the scanning domain for which initiation or growth stages are

359 not captured.

360 For these Fig. 9 demonstrations, it is observed that the local growth and/or decay

361 rates of the maximum reflectivity are typically less than $10 \mathrm{dBZ} /$ minute and exhibit no

362 clear relationship between the maximum reflectivity and its local rate of change for the

363 surrounding minute of radar observations (Figs. 9a-b). Median cloud reflectivity values

364 and the associated local rates of change are more gradual and demonstrate a maximum of

$3655 \mathrm{dBZ} /$ minute (Figs. 9c-d). Similarly, the local rate of change is likely to be independent 
366 of the median reflectivity value. In contrast to the maximum, the median value and its

367 relationship to its local rate of change is shown to be strongly tied to relative location of

368 the cloud element to the radar location, wherein lower magnitudes of the median are

369 observed closer to the radar location (Figs. 9b-d). This is an obvious consequence of

370 cloud elements having reduced radar sensitivity with range due to increased range gate

371 volume with distance from radar. The influence of radar sensitivity is larger if

372 considering the evolution of the mean cloud reflectivity and its local rate of change (not

373 shown). This indicates a limited relationship between these parameters and their rate of

374 change, thus showing the larger influence of radar sensitivity when analyzing the time

375 evolution of the mean and median cloud reflectivity.

376 


\section{Discussion and Conclusions}

This manuscript offers preliminary findings towards the potential capability to

379 track and document the lifecycle of shallow and weak cumulus to drizzling and showery

380 cumulus clouds using a scanning millimeter wavelength cloud radar. Whereas cloud

381 radars typically exhibit enhanced sensitivity for the detection of these low-level cloud

382 features, the ability of the Scanning ARM Cloud Radar (SACR) mm-wavelength radar

383 for detection of the particular shallow and non-precipitating boundary layer clouds from

384 this dataset was improved owing to a reduced signal-to-clutter ratio and suppressed

385 coherent scattering (e.g., Kollias et al., 2007). An AW-RHI scan strategy was

386 implemented during the MC3E campaign and included high temporal sampling to

387 facilitate the following of transient cloud elements as they advect with the mean wind

388 field over the SACR platform at the ARM Southern Great Plains - Central Facility (SGP

$389-\mathrm{CF}$ ). The ARM SGP site during the MC3E campaign was home to a network of

390 complementary scanning precipitation radars, lidar and collocated surface cloud

391 properties instrumentation that gave context to SACR observations. Simple

392 morphological analysis of complementary reflectivity factor observations from the

393 scanning radar facilities in particular helps demonstrate the potential benefits for having

394 multi-wavelength radar facilities of various scanning coverage scales, or cloud

395 observational 'supersites', to help bridge gaps between different cloud scales. There are

396 significant morphological implications when one is unable to capture the full dynamic

397 range of clouds from the smallest scales that may be detected by the millimeter

398 wavelength radar (SACR) to the larger scales covered by the centimeter radars (X- and

399 C-SAPR). 
To better demonstrate the capabilities of the ARM SACR systems for the 401 documentation of shallow cumulus evolution, a radar-based tracking algorithm (Cloud 402 Identification and Tracking Algorithm - CITA) was developed. A goal for CITA was to 403 explore the possibilities for a functional method to track key cloud microphysical and 404 geometric parameters, including their evolution in time and space, which are of interest to 405 detailed cloud process studies and cloud model evaluation. Basic sensitivity testing for 406 our initial set of CITA parameter outputs revealed that the current CITA design is capable 407 of reliably documenting cloud metrics, such as cloud element counts, maximum radar 408 reflectivity factor and cloud geometric properties including cloud top and cross-sectional 409 area. CITA was tested on a postfrontal shallow cumulus dataset collected by the SACR 410 when performing along-wind scans during MC3E on 25 May 2011. This day exhibited a 411 wide variety of cumulus cloud conditions and featured two and one half hours of 412 uninterrupted rapid radar scanning rates thereby allowing CITA to track clouds 413 unambiguously with time (e.g., Figs. 6, 7). The vast majority of the cloud elements 414 detected by CITA were short-lived with lifecycles shorter than 15 minutes, most of them 415 decaying after the first 5 minutes and exhibiting low maximal reflectivity cores.

416 Three long-lived cumulus clouds were captured during the collection period and 417 attained high reflectivity values that can be associated with precipitation onset in the 418 region. The associated time evolution captured by CITA is complex, yet potentially 419 highlights the eventual suffocation of the previously surface driven-sort shallow cumulus 420 clouds (albeit, those having additional larger-scale forcing in the post-frontal 421 environment) in the vicinity of the Ka-SACR. Moreover, observations suggest that these 422 deeper precipitation modes preceded sequences of higher-based non-precipitating 
423 cumulus cloud over the site, with these higher based clouds possibly influenced by

424 downward mixing of dry air associated with the preceding precipitation over or near the

425 region. Overall, most precipitation-free times demonstrate interesting behaviors during

426 the presented event, including bimodal or more complex distributions of low-level

427 cumulus clouds in terms of cloud-top heights and of smaller relative horizontal lengths.

428 Additional interpretation of the CITA dataset outputs in the context of the 25 May

4292011 MC3E event indicates most cloud elements reflect numerous shallow, non-

430 precipitating clouds having a maximum radar reflectivity lower than $-5 \mathrm{dBZ}$ (near the

431 traditional "first echo" limit of precipitation radars). These shallow cumuli were often

432 observed to be short-lived. The time-varying behaviors of the maximum and median

433 cloud reflectivity and local attempts to calculate associated rates of change for non-

434 precipitating shallow cumulus examples were less conclusive (e.g., Fig. 9). It is not

435 surprising to note that in following the evolution of median (and mean) cloud element

436 reflectivity factors, the tracking must account for changes in the sensitivity of the radar to

437 cloud echoes to be of much use. Nevertheless, following cloud maximum behaviors (less

438 influenced by radar sensitivity issues) as tractable quantities for microphysical evolution

439 of the clouds was also challenging to interpret, as echo maximums are found to evolve

440 quite rapidly and significantly in magnitude for well-captured shallow, non-precipitating

441 cloud echo elements and within only a few minutes of observation.

442 As this is the first application of CITA, more datasets are needed to drive a more

443 robust verification of the CITA methodology and to allow more comprehensive cloud

444 statistics. The findings for this study are also limited to shallow cloud observations from

445 the Oklahoma SGP ARM facility, although we anticipate the methods should translate 
446 well to other ARM facilities for similar low cloud conditions. Application of CITA in

447 real-time or field campaign settings is also however nontrivial and strongly tied to an

448 ability to characterize the cloud-level winds and appropriately (and repeatedly) target the

449 same cloud elements in time that are assumed to propagate along that mean wind

450 direction. Highly variable wind with time and, in the case of larger more vigorous clouds,

451 cloud development/decay alignment can play a role in a successful implementation of this

452 methodology as a fully automated tracking system. While those assumptions for most

453 cloud types are likely viable, tracking algorithm design problems may be exacerbated by

454 the narrow beamwidth of the Ka-SACR $\left(0.3^{\circ}\right)$ and other similar cloud radar systems.

455 Moreover, for such small beamwidths, only very small errors when establishing a mean

456 horizontal wind direction could affect substantial decreases in the quality and continuity

457 of the measurements, additional details on the sensitivity to the horizontal wind direction

458 is described in the appendix. Specifically, this suggest that several clouds would not

459 likely follow a path over the radar site and therefore represent an eventual inability for

460 the radar to track the complete (or best-case partial) evolution of valid cloud elements

461 with time. Different scanning strategies (including routine or reference sector scans) can

462 mitigate some of these known difficulties (e.g., Boundary Layer - RHIs, additional

463 details on scan strategies are described in Kollias et al. 2013a). However, utilizing these

464 scans implies a tradeoff between the scanning necessary for adequate temporal revisit of

465 cloud elements for tracking and microphysical process monitoring as compared to the

466 needs to assure the individual clouds are properly captured or tracked in full spatial

467 contexts. 


\section{Acknowledgements}

470 The authors wish to thank the reviewers whose insightful comments and

471 suggestions enhance this manuscript. This manuscript has been authored by employees of

472 Brookhaven Science Associates, LLC under Contract No. DE-AC02-98CH10886 with

473 the U.S. Department of Energy. The publisher by accepting the manuscript for 474 publication acknowledges that the United States Government retains a non-exclusive, 475 paid-up, irrevocable, world-wise license to publish or reproduce the published form of 476 this manuscript, or allow others to do so, for United States Government purposes. Dr. 477 Giangrande's work is supported by the U.S. Department of Energy, Office of Science, 478 Office of Biological and Environmental Research (OBER) as part of the ASR and ARM 479 programs. Additional author support was provided by the US DOE Atmospheric System 480 Research (ASR) program and by the BNL Laboratory Directed Research and 481 Development (LDRD) Program.

482

483

484

485

486

487

488

489

490

491 


\section{Appendix}

494 The success of CITA partially depends on the accurately determination of the

495 environment wind direction for the set up of the along-wind scan strategy (AW-RHI).

496 This is extremely dependent on the wind field and the cloud lifetime. In this particular

497 case, the wind field is not expected to significantly influence the results since it did not

498 present an appreciable change in time or height and there was no distinguishable shear

499 that could cause clouds to move differently at different heights. Considering a very

500 simple advection model the deviation from the wind direction that the AW-RHI scan

501 strategy can have and still sample the same volume will mostly depend on the size of the

502 region that is assumed homogeneous, the wind speed at which clouds propagate and

503 cloud lifecycle. Due to the Ka-SACR beamwidth at a $10 \mathrm{~km}$ distance from the radar the

504 sampling volume is approximately a cylinder of $\sim 50 \mathrm{~m}$ diameter, considering this to be

505 the size of the region to be homogeneous, assuming a constant horizontal wind and that

506 cloud elements are advected over the radar domain with the middle point of their

507 lifecycle occurring over the location of the radar then the estimation of the maximum

508 deviation of the radar scan angle from the wind direction can be estimated (Fig. 10).

509 Small errors in the horizontal wind direction could substantially impact the use of this

510 technique, for this particular case study, considering wind speeds slower than $20 \mathrm{~m} / \mathrm{s}$

511 (Fig. 2) and cloud lifetime shorter than 10 minutes (Fig. 6), the same cloud volume will

512 be sampled approximately 20 times and capture the evolution of the same parcel only if

513 there is a variation of 1 degree between the scan angle and the wind direction. However,

514 considering a slower advection speed of $10 \mathrm{~m} / \mathrm{s}$ and shorter cloud lifecycles then the 
515 disagreement between the cloud propagating and scan angle can be close to 5 degrees and 516 still sample proprieties within the same volume, and of course considering a larger parcel 517 size will also modify these results by allowing a larger disagreement between the angles

518 (i.e., if assuming a homogeneous volume with diameter of $100 \mathrm{~m}$, the deviation between 519 the angles can be almost doubled).

520

521

522 


\section{6. REFERENCES}

524

525 Ackerman, T. A., and G. M. Stokes, 2003: The Atmospheric Radiation Measurement

526 Program. Phys. Today, 56, 38-45.

527

528 Boer, E., and V. Ramanathan, 1997: Lagrangian approach for deriving cloud

529 characteristics from satellite observations and its implications to cloud parameterizations.

530 J. Geophys Res., 102, 21383-21399

531

532 Burnet, F., and J.-L. Brenguier, 2010, The onset of precipitation in warm cumulus clouds:

533 An observational case-study. Q.J.R. Meteorol. Soc., 136, 374-381. doi: 10.1002/qj.552

534

535 Chen, S. S., and B. E. Mapes, 1996: Multiscale variability of deep convection in relation

536 to large-scale circulation in TOGA COARE. J. Atmos. Sci., 53, 1380-1409.

537

538 Dixon, M., and G. Wiener, 1993: TITAN: Thunderstorm Identification, Tracking,

539 Analysis, and Nowcasting-A radar-based methodology. J. Atmos. Oceanic Technol., 10, $540 \quad 785-797$.

541

542 Feingold, G., and H. Siebert, 2009: In Clouds in the Perturbed Climate System: Their

543 Relationship to Energy Balance, Atmospheric Dynamics, and Precipitation. J.

544 Heintzenberg, and R. J. Charlson, eds., Strüngmann Forum Reports, 2, The MIT Press, 545597 pp. 
547 French, J. R., G. Vali, and R. D. Kelly, 1999: Evolution of small cumulus clouds in

548 Florida: Observations of pulsating growth. Atmos. Res, 52:143-165.

549

550 Futyan, J. M., and A. D. Del Genio, 2007: Deep Convective System Evolution over

551 Africa and the Tropical Atlantic. J. Climate, 20, 5041-5060.

552

553 Ghan, S. J., L. R. Leung, J, and McCaa, 1999: A Comparison of Three Different

554 Modeling Strategies for Evaluating Cloud and Radiation Parameterizations. Mon. Wea.

555 Rev., 127, 1967-1984.

556

557 Giangrande, S.E., S. Collis, A. Theisen, and A. Tokay, 2013; Precipitation Estimation

558 from the ARM Distributed Radar Network During the MC3E Campaign. Submitted, J.

559 Appl. Meteorol. and Climatol.

560

561 Göke, S., H. T. Ochs, and R. M. Rauber, 2007: Radar Analysis of Precipitation Initiation

562 in Maritime versus Continental Clouds near the Florida Coast: Inferences Concerning the

563 Role of CCN and Giant Nuclei. J. Atmos. Sci., 64, 3695-3707.

564

565 Grenier, H., and C. S. Bretherton, 2001: A Moist PBL Parameterization for Large-Scale

566 Models and Its Application to Subtropical Cloud-Topped Marine Boundary Layers. Mon.

567 Wea. Rev., 129, 357-377.

568 
569 Illingworth, A. J., R. J. Hogan, E. J. O'Connor, D. Bouniol, M. E. Brooks, J. Delanoe, D.

570 P. Donovan, J. D. Eastment, N. Gaussiat, J. W. F. Goddard, M. Haeffelin, H. Klein

571 Baltink, O. A. Krasnov, J. Pelon, J.-M. Piriou, A. Protat, H. W. J. Russchenberg, A.

572 Seifert, A. M. Tompkins, G.-J. van Zadelhoff, F. Vinit, U. Willen, D. R. Wilson, and C.

573 L. Wrench, 2007. Bull. Am. Meteorol. Soc., 88, 883-898.

574

575 Johnson, J. T., P. L. MacKeen, A. Witt, E. D. Mitchell, G. J. Stumpf, M. D. Eilts, and K.

576 W. Thomas, 1998: The storm cell identification and tracking algorithm: An enhanced

577 WSR-88D algorithm. Wea. Forecasting, 13, 263-276.

578

579 Jorgensen, D. P., E. J. Zipser, M. A. LeMone, 1985: Vertical Motions in Intense 580 Hurricanes. J. Atmos. Sci., 42, 839-856.

581

582 Knight, C. A., and L. J. Miller, 1993: First radar echoes from cumulus clouds. Bull.

583 Amer. Meteor. Soc., 74, 1179-1193

584

585 Knight, C. A., and L. J. Miller, 1998: Early radar echoes from small, warm cumulus:

586 Bragg and hydrometeor scattering J. Atmos. Sci., 55,2974-2992

587

588 Knight, C. A., J. Vivekanandan, and S. G. Lasher-Trapp, 2002: First Radar Echoes and

589 the Early ZDR History of Florida Cumulus. J.Atmos. Sci., 59, 1454-1472

590 
591 Kollias P., N. Bharadwaj, K. Widener, I. Jo, and K. Johnson, 2013a: Scanning ARM

592 Cloud Radars - Part I: Operational Sampling Strategies. In review to J. Atmos. Oceanic

593 Technology.

594

595 Kollias P., I. Jo, P. Borque, A. Tatarevic, K. Lamer, N. Bharadwaj, K. Widener, K.

596 Johnson, and E. E. Clothiaux, 2013b: Scanning ARM Cloud Radars - Part II: Data

597 Quality Control and Processing. In review to J. Atmos. Oceanic Technology.

598

599 Kollias, P., E. E. Clothiaux, A. A. Miller, B. A. Albrecht, G. L. Stephens, and

600 T.P. Ackerman, 2007: Millimeter-wavelength radars: New frontier in atmospheric cloud 601 and precipitation research. Bull. Amer. Meteo. Soc., 88: 1608-1624. doi: 10.1175/BAMS$60288-10-1608$.

603

604 Lu, M.-L., G. Feingold, H. H. Jonsson, P. Y. Chuang, H. Gates, R. C. Flagan, and J. H. 605 Seinfeld, 2008, Aerosol-cloud relationships in continental shallow cumulus, J. Geophys. 606 Res., 113, D15201, doi:10.1029/2007JD009354.

607

608 Machado, L. A., W. B. Rossow, R. L. Guedes, and A. W. Walker, 1998: Life cycle

609 variations of mesoscale convective systems over the Americas. Mon. Wea. Rev., 126,

$610 \quad 1630-1654$.

611

612 Maddox, R. A., 1980: Mesoscale convective complexes. Bull. Amer. Meteor. Soc., 61, $613 \quad 1374-1387$. 
615 Mather, J. H., and J. W. Voyles, 2013: The Arm Climate Research Facility: A Review of

616 Structure and Capabilities. Bull. Amer. Meteor. Soc., 94, 377-392.

617 doi: http://dx.doi.org/10.1175/BAMS-D-11-00218.1

618

619 Park, S., and C. S. Bretherton, 2009: The University of Washington Shallow Convection 620 and Moist Turbulence Schemes and Their Impact on Climate Simulations with the 621 Community Atmosphere Model. J. Climate, 22, 3449-3469.

622

623 Rosenfeld, D., 1987: Objective method for analysis and tracking of convective cells as 624 seen by radar. J. Atmos. Oceanic Technol., 4, 422-434.

625

626 Stephens, G. L., 2005: Cloud Feedbacks in the Climate System: A Critical Review. J.

627 Climate, 18, 237-273. doi: http://dx.doi.org/10.1175/JCLI-3243.1

629 Stevens, B., and G. Feingold, 2009: Untangling aerosol effects on clouds and 630 precipitation in a buffered system, Nature 461, 607-613

631

632 Stokes, G. M., and S. E. Schwartz, 1994: The Atmospheric Radiation Measurement 633 (ARM) Program: Programmatic background and design of the cloud and radiation 634 testbed. Bull. Amer. Meteor. Soc., 75, 1201-1221.

635 
636 Velasco, I., and J. M. Fritsch, 1987: Mesoscale convective complexes in the Americas. $J$.

637 Geophys. Res., 92, 9591-9613.

638

639 Williams, M., and R. A. Houze, 1987: Satellite-observed characteristics of winter 640 monsoon cloud clusters. Mon. Wea. Rev., 115, 505-519.

641 


\begin{tabular}{|l|l|}
\hline \multicolumn{2}{|c|}{ Scanning ARM Cloud Radar (Ka-band) } \\
\hline Scan type & Along-Wind Horizon-to-Horizon \\
\hline Nyquist velocity & $10.5 \mathrm{~ms}^{-1}$ \\
\hline Range resolution & $20 \mathrm{~m}$ \\
\hline Scan time & $\sim 30 \mathrm{sec}$ \\
\hline PRF & $5 \mathrm{kHz}$ \\
\hline Sensitivity & $\sim-30 \mathrm{dBZ}$ at $10 \mathrm{~km}$ \\
\hline Frequency & $35.29 \mathrm{GHz}$ \\
\hline Wavelength & $8.5 \mathrm{~mm}$ \\
\hline
\end{tabular}

643 Table 1: Ka-band Scanning ARM Cloud Radar (Ka-SACR) technical specifications

644

645 
648 Figure 1. Map showing the heterogeneous ARM radar network at the SGP facility. Blue 649 rings indicate a $20 \mathrm{~km}$ radius around each X-band radar and red ring indicates a $30 \mathrm{~km}$ 650 radius around the C-band radar.

651

652 Figure 2. Wind magnitude (a) and direction (b) from radiosonde observations at SGP 653 CF at 1730 UTC (blue) and 2030 UTC (black) on 25 May 2011.

655 Figure 3. Hemispherical view of the cloud field at the Central Facility from the Total Sky 656 Imager (a), reflectivity from the C-SAPR at 2011 UTC (b), X-SAPR at 2010 UTC(c) PPI 657 scan at $1.2^{\circ}$ and $1.5^{\circ}$ respectably and from Ka-SACR AW-RHI scan at 2010 UTC (d) on 65825 May 2011 when weak non-precipitating cloud were present over the Central Facility. 659 Blue triangle represents the location of the radar and white dot represents the location of 660 the Central Facility; on panels (b) and (c) black line represents the SACR scan and black 661 circle represents the domain where SE X-SAPR data are collected. Orientation in panel d) 662 is NW on the right and SE on the left.

664 Figure 4. Reflectivity from three consecutive Ka-SACR along-wind scans from 19:44 to 665 19:45 UTC (shaded) and cloud's identification number documented by the CITA 666 (Contour). Radar location is depicted by the yellow rectangle and the time of each scan is 667 indicated in the bottom left sector of each sub-panel. 
669 Figure 5. Maximum reflectivity frequency (bin: $2 \mathrm{~dB}$, upper panel) and cloud area cross-

670 section frequency (bin: $0.5 \mathrm{~km} 2$, lower panel) for different reflectivity detections

671 threshold in the Cloud Identification and Tracking Algorithm for the 25th May 2011 case.

672

673 Figure 6. Cross-sectional area (a) and maximum reflectivity (b) as a function of time for 674 every element detected by the CITA on 25 May 2011 during the 2.5-hour window. Colors 675 represent individual clouds tracked by CITA.

676

677 Figure 7. Histogram of maximum reflectivity (a) and area (b) of all cloud elements 678 detected by CITA for the 25 May 2011 case.

679

680 Figure 8. A sequence of TSI images during the 2.5-hour long observing period (a), the 681 number of cloud elements observed in the Ka-SACR AW-RHI scans as a function of time 682 (b), the histogram of detected maximum cloud horizontal length from the Ka-SACR as a 683 function of time (c), the histogram of detected cloud top heights from the Ka-SACR as a 684 function of time (d), and number of drops registered by the ARM disdrometer (e). 685

686 Figure 9. Temporal evolution of maximum reflectivity for shallow cumuli (a) and the rate 687 of change of maximum reflectivity (b) using a one-minute averaging window as a 688 function of the mean maximum reflectivity over the segment where the rate of change 689 was computed for small shallow cumulus clouds over SGP on May 25, 2011. Respective 690 calculations for median reflectivity are shown in panels (c) and (d). For (b) and (d) color 
691 code indicates the maximum distance between the cloud element outer edge and the radar 692 location $[\mathrm{km}]$.

693

694 Figure 10. Maximum deviation of the radar scan angle from the wind direction so 695 that the radar measures variables within a homogeneous volume of $50 \mathrm{~m}$ diameter 696 as a function of wind speed and cloud lifetime. The area in the top right corner 697 delimitated by the black thick line represents the region where clouds cannot be observed 698 given their time required to sample their full lifecycle, the wind speed, and the domain 699 size.

700

701 


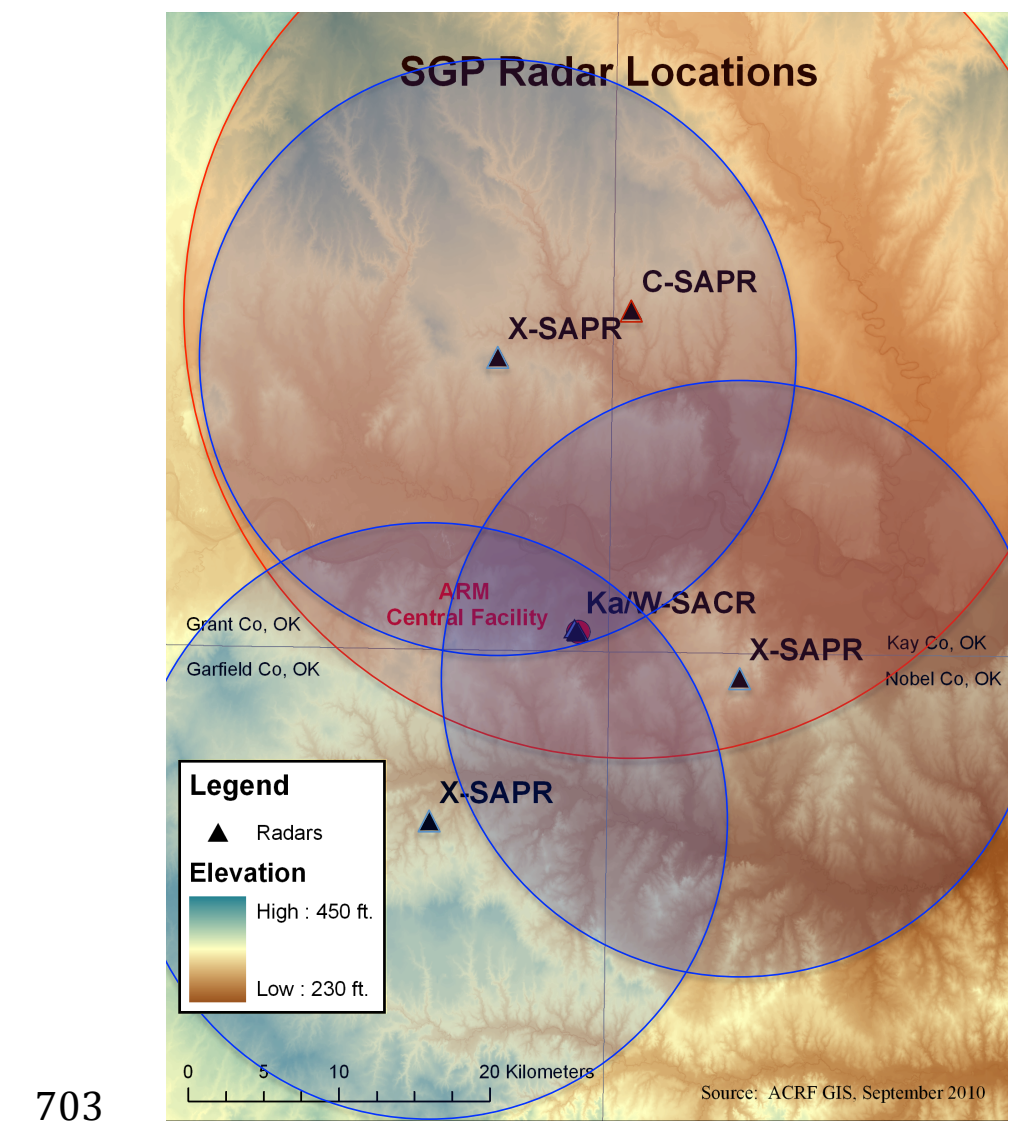

704 Figure 1. Map showing the heterogeneous ARM radar network at the SGP facility. Blue

705 rings indicate a $20 \mathrm{~km}$ radius around each X-band radar and red ring indicates a $30 \mathrm{~km}$

706 radius around the $\mathrm{C}-$ band radar. 

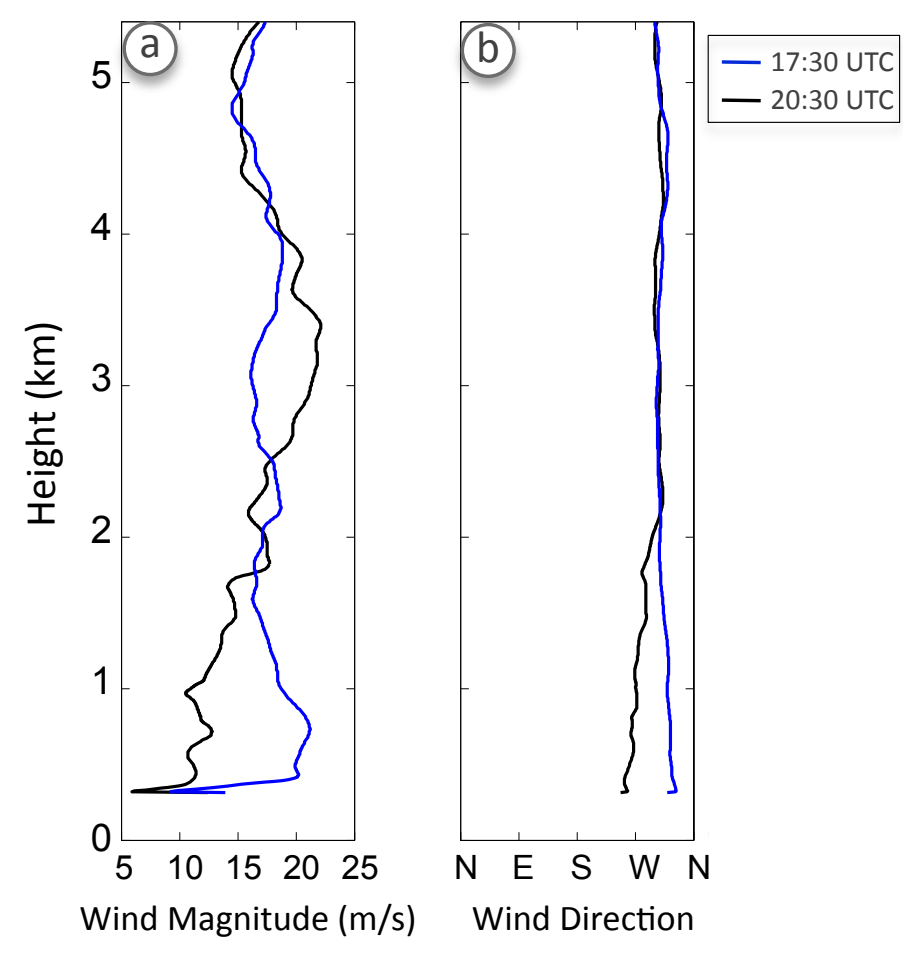

708 Wind Magnitude $(\mathrm{m} / \mathrm{s})$

709 Figure 2. Wind magnitude (a) and direction (b) from radiosonde observations at SGP -

710 CF at 1730 UTC (blue) and 2030 UTC (black) on 25 May 2011.

711

712 

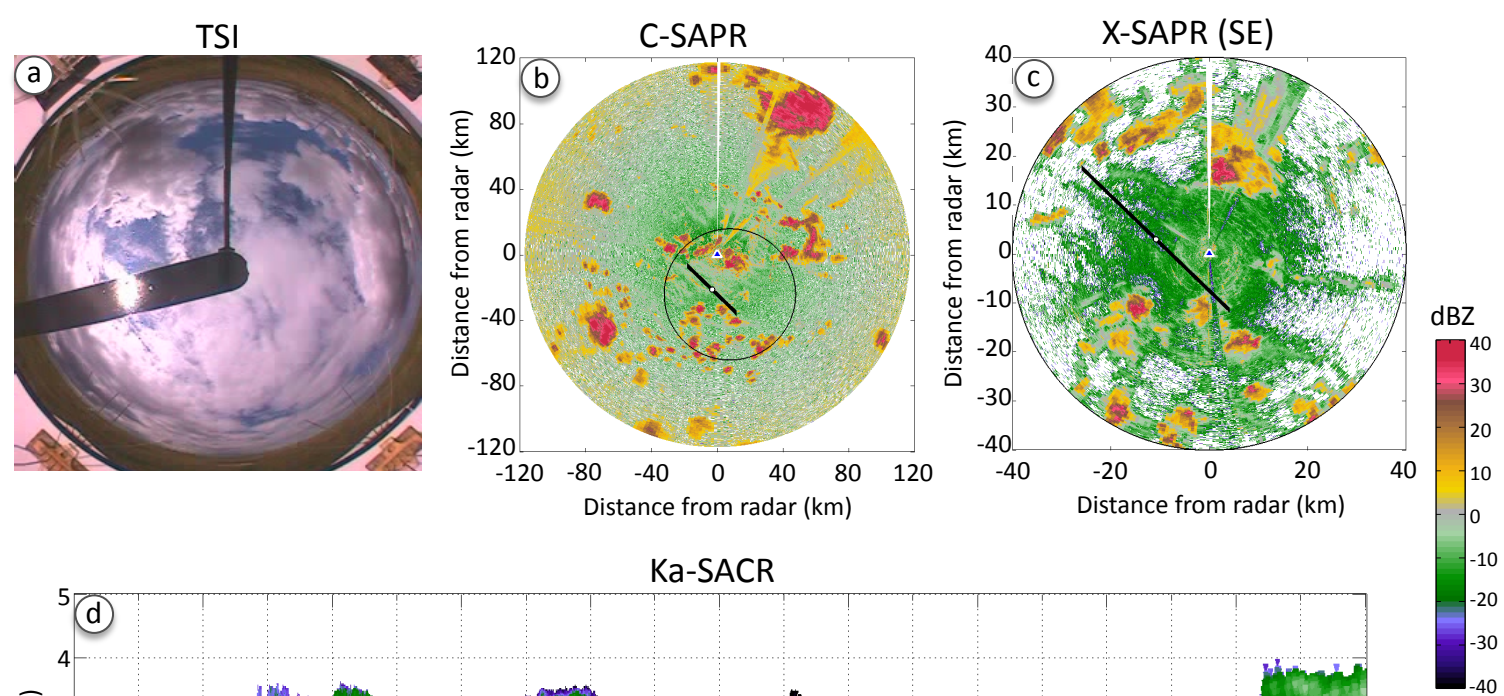

713

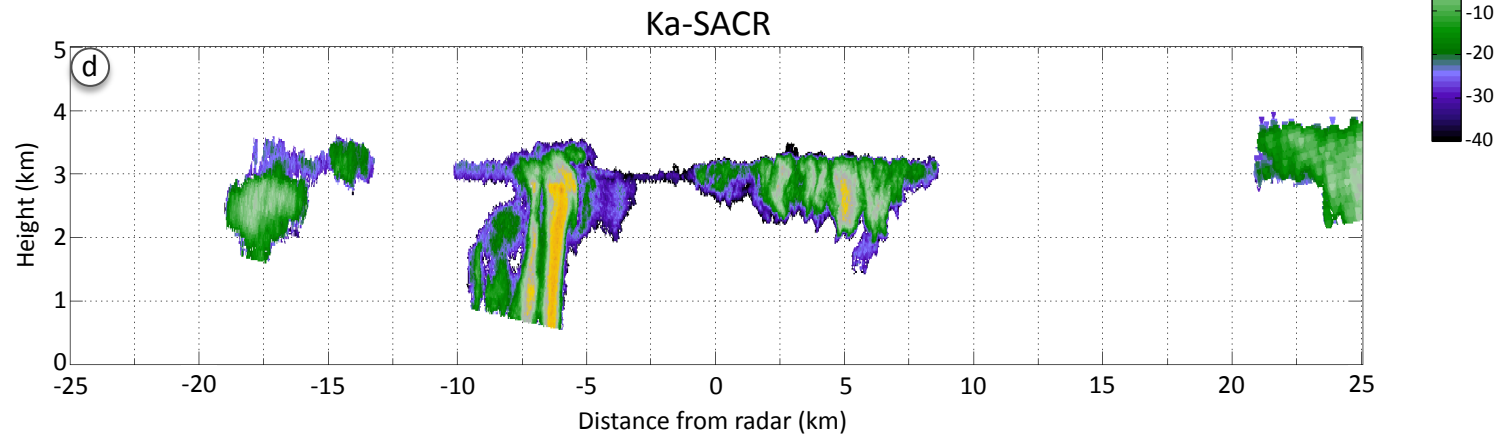

714 Figure 3. Hemispherical view of the cloud field at the Central Facility from the Total Sky

715 Imager (a), reflectivity from the C-SAPR at 2011 UTC (b), X-SAPR at 2010 UTC(c) PPI

716 scan at $1.2^{\circ}$ and $1.5^{\circ}$ respectably and from Ka-SACR AW-RHI scan at 2010 UTC (d) on

71725 May 2011 when weak non-precipitating cloud were present over the Central Facility.

718 Blue triangle represents the location of the radar and white dot represents the location of

719 the Central Facility; on panels (b) and (c) black line represents the SACR scan and black

720 circle represents the domain where SE X-SAPR data are collected. Orientation in panel

721 (d) is NW on the right and SE on the left.

722

723

724

725 


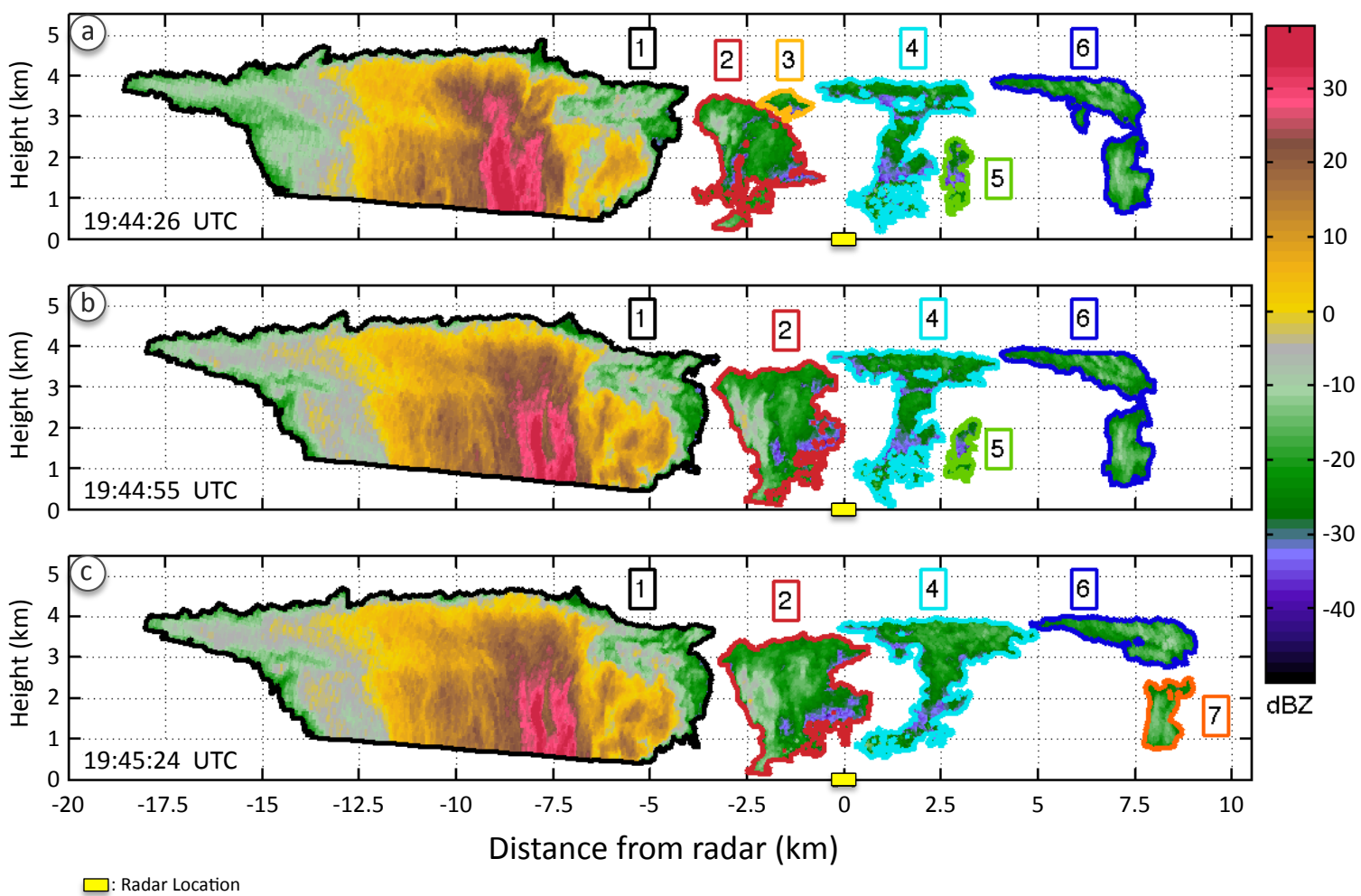

727 Figure 4. Reflectivity from three consecutive Ka-SACR along-wind scans from 19:44 to

728 19:45 UTC (shaded) and cloud's identification number documented by the CITA

729 (Contour). Radar location is depicted by the yellow rectangle and the time of each scan is

730 indicated in the bottom left sector of each sub-panel. 

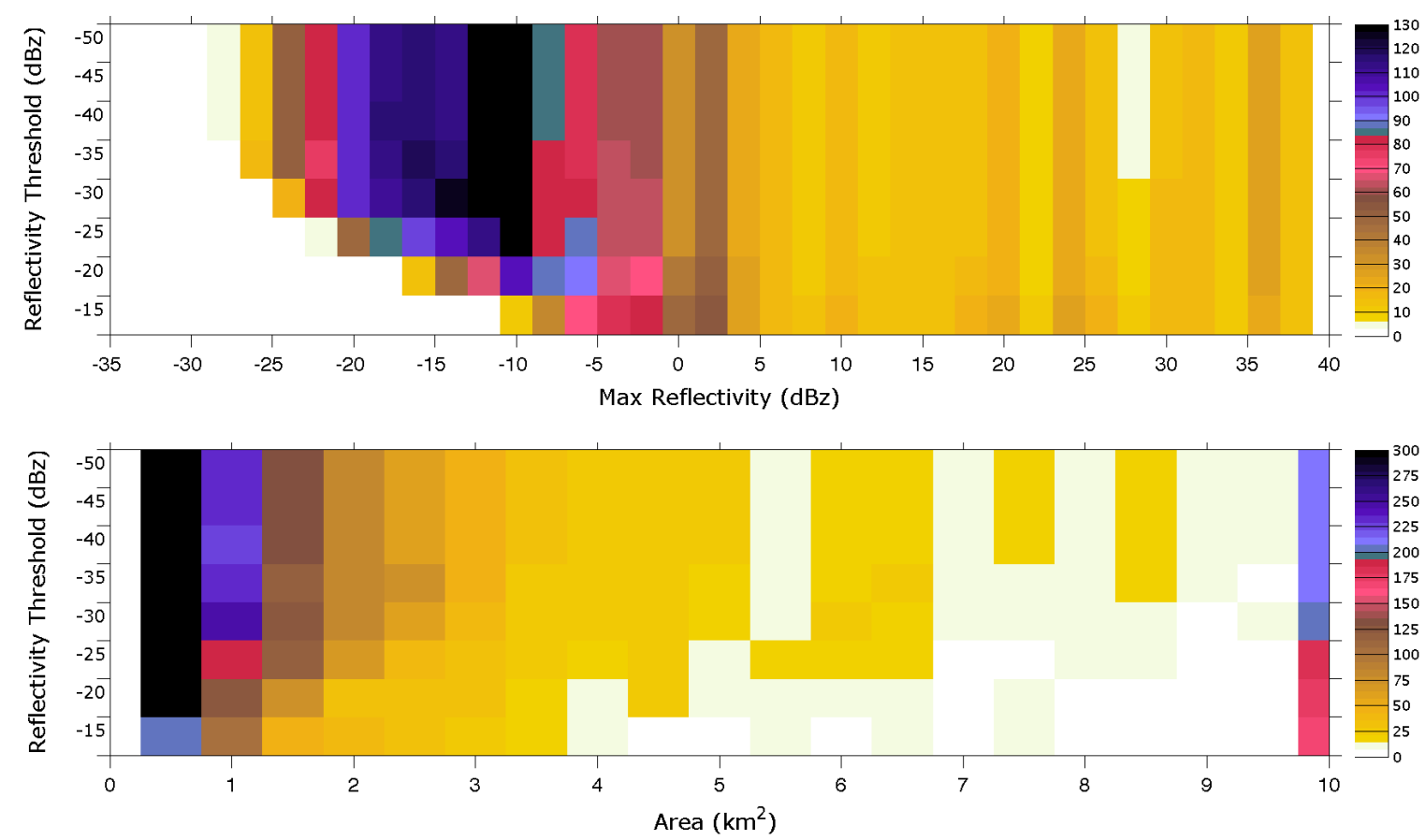

736 Figure 5. Maximum reflectivity frequency (bin: $2 \mathrm{~dB}$, upper panel) and cloud area cross-

737 section frequency (bin: $0.5 \mathrm{~km} 2$, lower panel) for different reflectivity detections

738 threshold in the Cloud Identification and Tracking Algorithm for the 25th May 2011 case. 

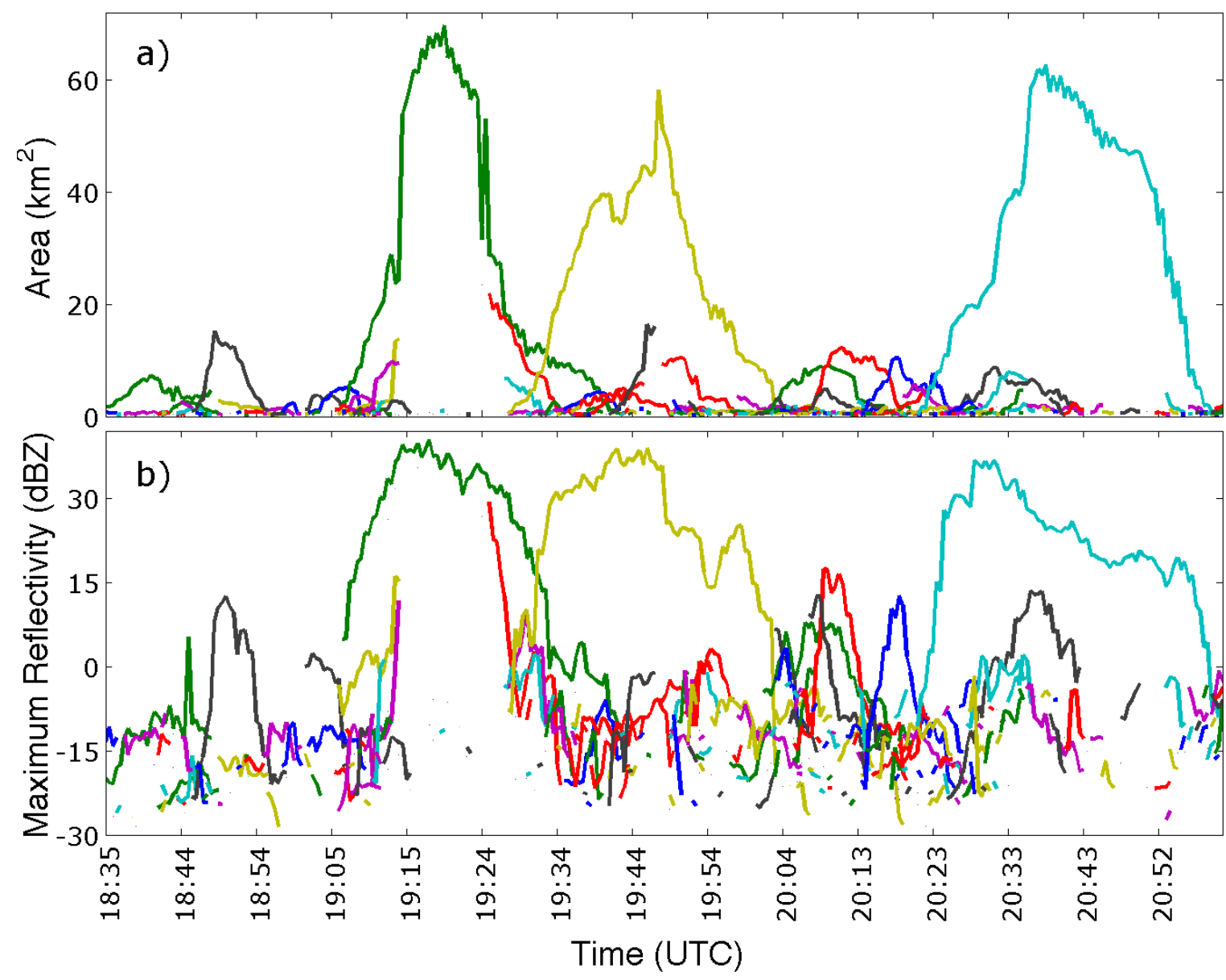

744 Figure 6. Cross-sectional area (a) and maximum reflectivity (b) as a function of time for

745 every element detected by the CITA on 25 May 2011 during the 2.5-hour window. Colors

746 represent individual clouds tracked by CITA. 

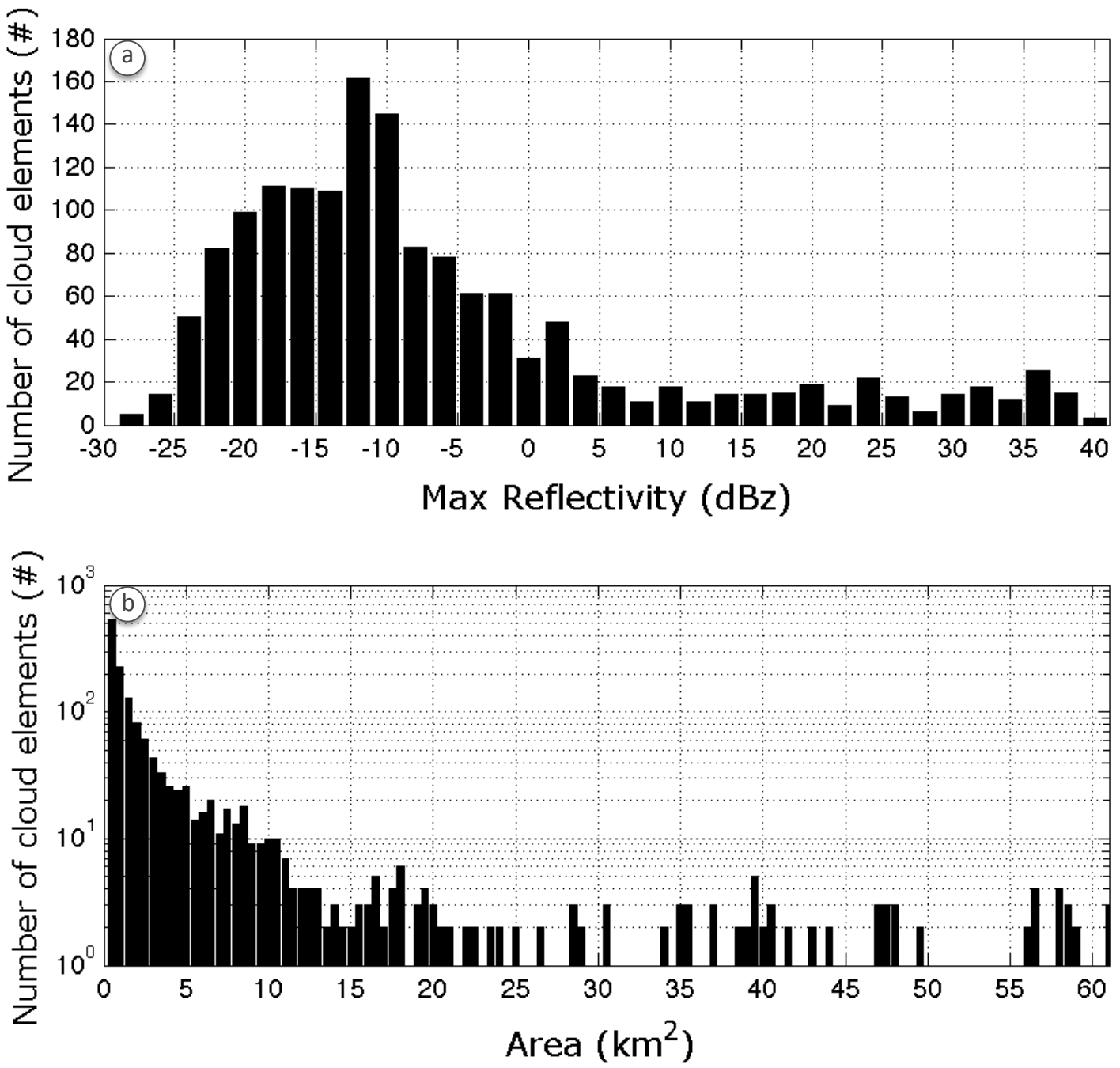

756 Figure 7. Histogram of maximum reflectivity (a) and area (b) of all cloud elements

757 detected by CITA for the 25 May 2011 case. 

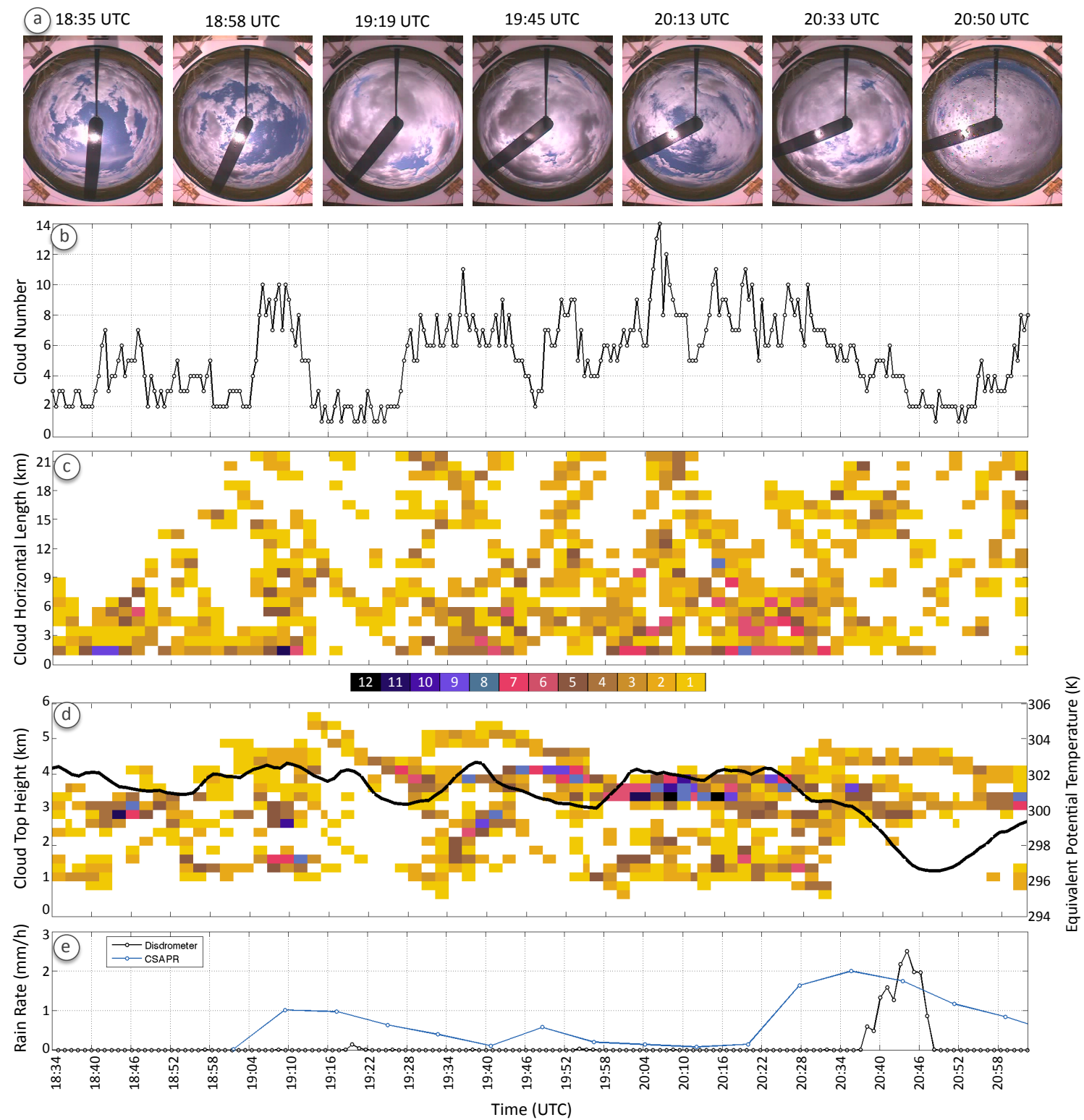

765 Figure 8. A sequence of TSI images during the 2.5-hour long observing period (a), the

766 number of cloud elements observed in the Ka-SACR AW-RHI scans as a function of time

767 (b), the histogram of detected maximum cloud horizontal length from the Ka-SACR as a

768 function of time (c), the histogram of detected cloud top heights from the Ka-SACR and

769 equivalent potential temperature as a function of time (d), and rain rate estimations from

770 the CSAPR and the ARM disdrometer (e). 

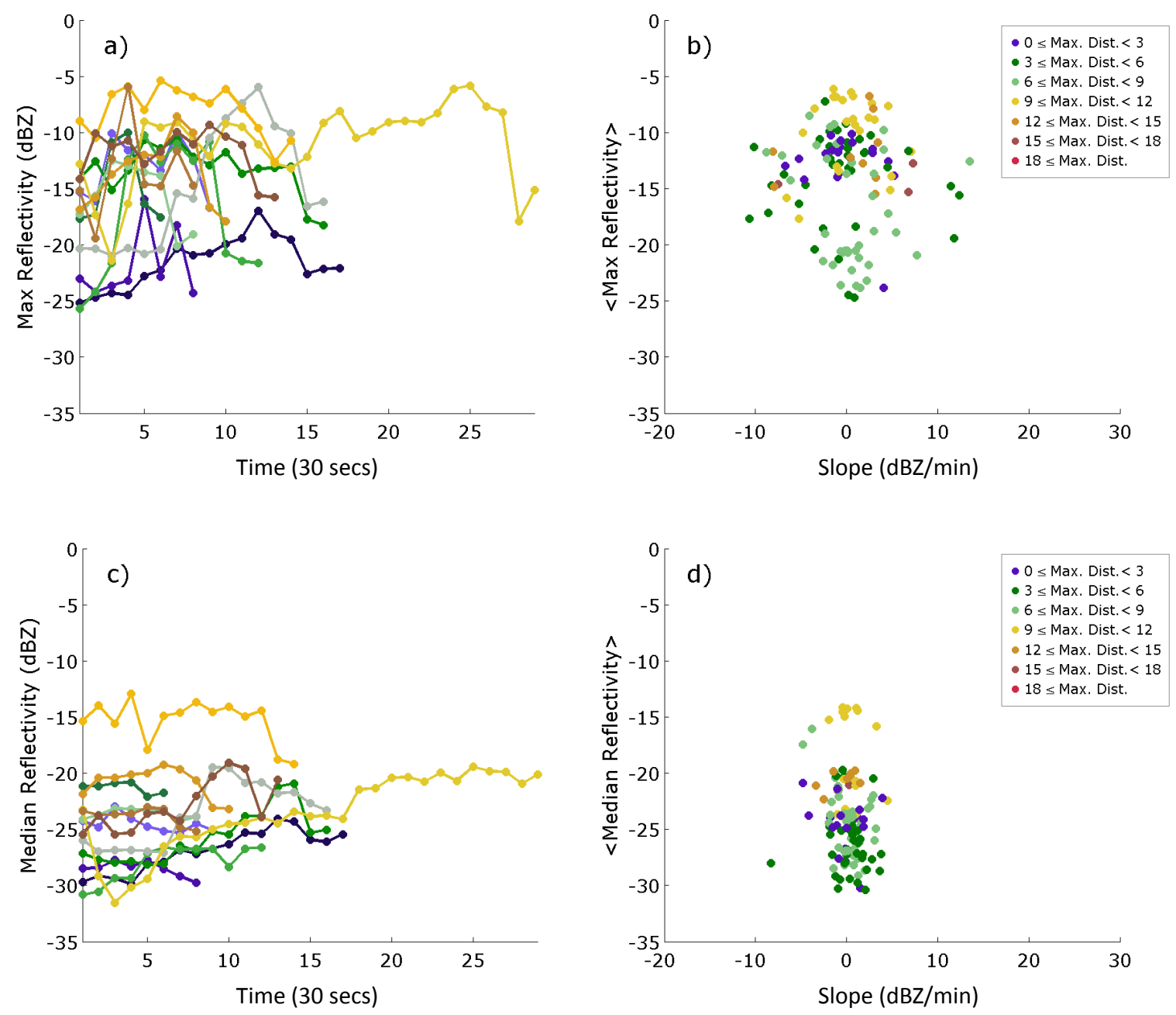

775 Figure 9. Temporal evolution of maximum reflectivity for shallow cumuli (a) and the rate

776 of change of maximum reflectivity (b) using a one-minute averaging window as a

777 function of the mean maximum reflectivity over the segment where the rate of change

778 was computed for small shallow cumulus clouds over SGP on May 25, 2011. Respective

779 calculations for median reflectivity are shown in panels (c) and (d). For (b) and (d) color

780 code indicates the maximum distance between the cloud element outer edge and the radar

781 location $[\mathrm{km}]$. 

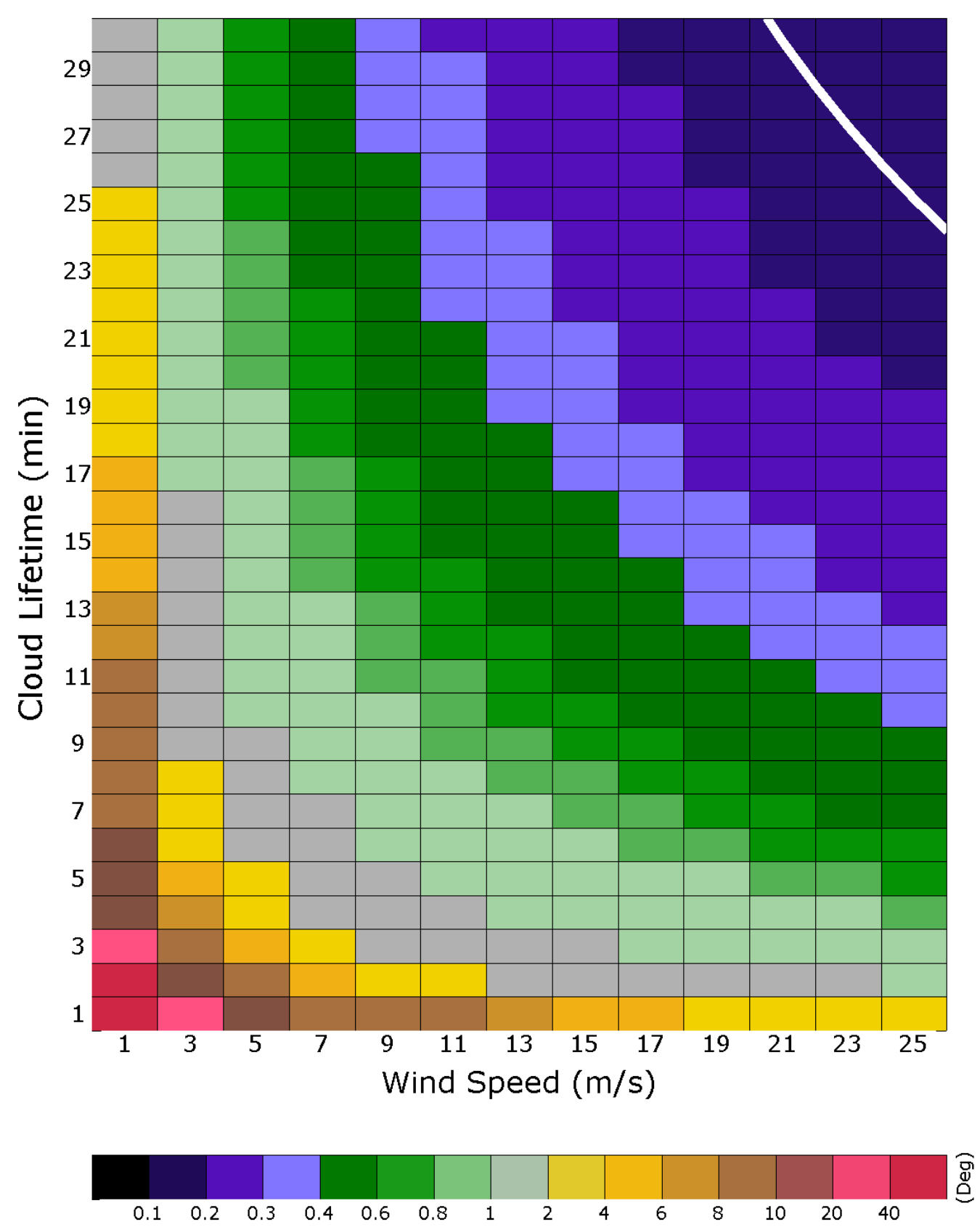

784 Figure 10. Maximum deviation of the radar scan angle from the wind direction so

785 that the radar measures variables within a homogeneous volume of $50 \mathrm{~m}$ diameter

786 as a function of wind speed and cloud lifetime. The area in the top right corner

787 delimitated by the white thick line represents the region where clouds cannot be observed

788 given their time required to sample their full lifecycle, the wind speed, and the domain 789 size. 